\title{
Grundlegende Betrachtungen zu kapazitiven Rußsensoren
}

\author{
Gunter Hagen, Robin Werner, Markus Feulner, Andreas Müller, Michael Schubert, Ralf Moos \\ Bayreuth Engine Research Center (BERC), Lehrstuhl für Funktionsmaterialien \\ Universität Bayreuth, 95440 Bayreuth
}

\section{Zusammenfassung}

Um kleinste Rußkonzentrationen im automobilen Abgas detektieren zu können, wird ein kapazitiver Messansatz vorgeschlagen. Die Finger-Elektroden eines nach dem Stand der Technik üblichen resistiven Rußsensors werden dafür mit einer sehr dünnen aber hochdichten $\mathrm{Al}_{2} \mathrm{O}_{3}$-Schicht isoliert. Die Grundüberlegung, dass eine Belegung dieser Oberfläche mit elektrisch leitfähigen Rußpartikeln zu einer Kapazitätserhöhung führt, kann sowohl modellhaft beschrieben als auch in Messungen im Diesel-Abgas nachgewiesen werden. Steigende Rußkonzentrationen führen im Vergleich zum resistiven Sensor zu einem schnelleren Ansteigen der Kapazität während eines Messzyklus.

Keywords: Rußsensoren, On-Board-Diagnose, COMSOLMultiphysics, Aerosol-Deposition, Motorprüfstand

\section{Einleitung und Motivation}

Strenge Vorgaben der Gesetzgeber erfordern neue Abgasnachbehandlungskonzepte und genaue Sensoren, um einen effizienten Fahrbetrieb und eine ständige Überwachung der Funktionalität aller Systeme (On-BoardDiagnose, OBD) zu gewährleisten. Bei DieselFahrzeugen ist der Einsatz von DieselPartikelfiltern (DPF) unumgänglich. Ein Rußsensor, der nach einem DPF verbaut ist, soll im Fehlerfall durch den Filter tretenden Ruß detektieren [1]. Die Empfindlichkeit eines solchen Sensors muss sehr hoch sein, um auch kleinste Rußmengen schnell und sicher erfassen zu können.

Typische bestehende Rußsensoren arbeiten nach dem resistiven Prinzip [2]. Dazu werden zwei voneinander getrennte Elektroden (Interdigitalstruktur, zumeist siebgedruckt aufgebbracht) auf einem planaren Keramiksubstrat hergestellt. Wird nun an den Elektroden eine elektrische Spannung angelegt und lagert sich elektrisch leitfähiger Ruß zwischen diesen ab, so kann ein Stromfluss detektiert werden, sobald sich über die Rußpartikel ein erster leitfähiger Pfad ausgebildet hat. Die Zeit bis zur Perkolation der Rußpartikel hängt vom Partikelgehalt bzw. der Partikelmasse im Gasstrom ab. Alternativ kann auch der Anstieg der Stromkurve über der Zeit als Maß für die Partikelkonzentration ausgewertet werden [3]. Die Höhe der anliegenden Spannung beeinflusst die Anlagerung von Rußteilchen zwischen den
Elektroden durch elektrophoretische Effekte [4].

\section{Grundüberlegung und theoretische Betrachtung}

Um die Ansprechzeit eines Rußsensors erhöhen zu können, wurde eine kapazitive Messung vorgeschlagen [5]. Dabei werden die interdigitalen planaren Elektroden mit einer elektrisch isolierenden Schicht abgedeckt (Abbildung 1).

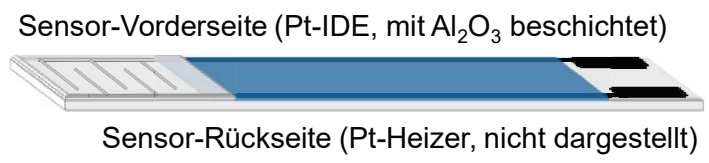

Abb. 1: Schematischer Sensor-Aufbau auf einem $\mathrm{Al}_{2} \mathrm{O}_{3}$-Substrat: Auf der SensorVorderseite befinden sich siebgedruckte Pt-Interdigital-Elektroden, die mit einer dünnen $\mathrm{Al}_{2} \mathrm{O}_{3}$-Schicht abgedeckt sind, auf der SensorRückseite befindet sich ein Dickschicht-Heizelement (nicht dargestellt), das auch als Temperatursensor dienen kann.

Sich anlagernder Ruß hat also keinen direkten Kontakt zu den Elektroden. Als Messgröße dient hier die Kapazität des Bauteils. Da die Feldlinien durch die leitfähigen Partikel "verkürzt" werden, muss ein Anstieg der Kapazität gemessen werden. Dieser Effekt 
sollte sich auch schon vor Erreichen der Perkolation der Rußpartikel bemerkbar machen.

Obige Überlegung bestätigt einfache Simulationen (Abbildung 2). Es wurde dazu der Feldverlauf im Bereich des Abstandes (Breite $100 \mu \mathrm{m})$ zwischen zwei halben Elektroden (Breite jeweils $50 \mu \mathrm{m}$ ) ohne und mit lokaler leitfähiger Bedeckung der isolierenden Schicht berechnet.

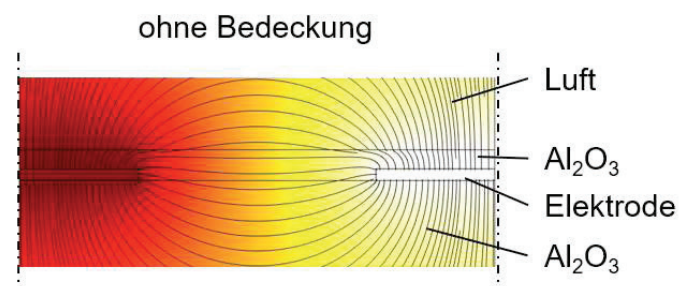

a)

b)

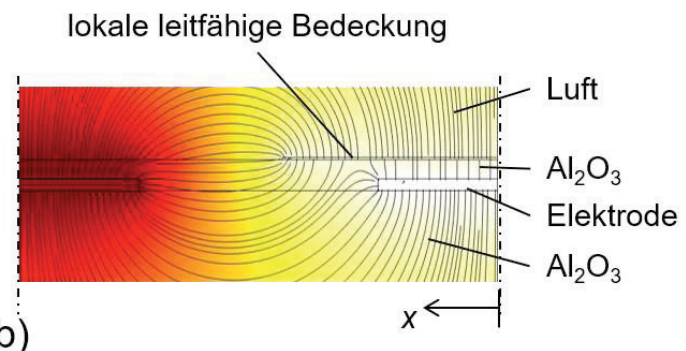

Abb. 2: Simulation der Feldverteilung im Bereich zwischen zwei interdigitalen Fingerelektroden mit COMSOL Multiphysics):

a) Die Feldlinien verlaufen symmetrisch im "unbeladenen" Fall. b) Sobald eine lokale leitfähige Bedeckung auf der Isolationsschicht über den Elektroden vorhanden ist, ändert sich der Verlauf der elektrischen Feldlinien

Man erkennt leicht, dass bei lokaler leitfähiger Bedeckung die Feldlinien nicht mehr symmetrisch wie im unbeladenen Fall verlaufen. Durch den elektrisch leitfähigen Ruß entsteht eine Äquipotentialschicht auf der Isolationsschicht. Hier stehen die Feldlinien senkrecht und der effektive Abstand zwischen den Elektroden wird verkürzt.

\section{Simulation der Kapazitätsänderung}

Wertet man nun die berechnete Oberflächenladungsdichte für den jeweiligen Feldverlauf und damit die Ladung auf den Elektroden aus und bezieht diese dann auf die komplette Elektrodenstruktur unter Berücksichtigung des Potentials, so kann man in Abhängigkeit der Breite des bedeckenden leitfähigen Materials einen Anstieg der Kapazität bis auf ein Sättigungsniveau (bei vollständiger Bedeckung) erkennen. Dabei gibt es einen Unterschied, ob die leitfähige Schicht von einer Seite ausgehend den Abstand der Elektroden überspannt (Fall 1), oder ob man annimmt, dass die Breite des leitfähigen Materials von der Mitte der Elektroden ausgehend vergrößert wird (Fall 2). Bei beiden Rechnungen wird deutlich, dass sich auch schon bei geringer Bedeckung (Breite $<50 \mu \mathrm{m})$ ein kleiner Kapazitätsanstieg einstellt. Eine starke Änderung tritt dann ein, wenn die leitfähige Schicht in den Bereich der Elektroden kommt: für den 2. Fall ist dies bei $100 \mu \mathrm{m}$ erkennbar, denn dann wird der gesamte Abstandsbereich zwischen den Elektroden überspannt; für den 1. Fall erkennt man den steilen Anstieg ab $150 \mu$ m Breite des leitfähigen Materials, da in der Berechnung hier eine der Elektroden und der Elektrodenabstand überspannt ist. Beide Rechnungen führen schließlich zu einer Sättigungskapazität für die vollständige Bedeckung der isolierenden Schicht, die für die angenommene Geometrie bei ca. 50 pF liegt (Abbildung 3a).
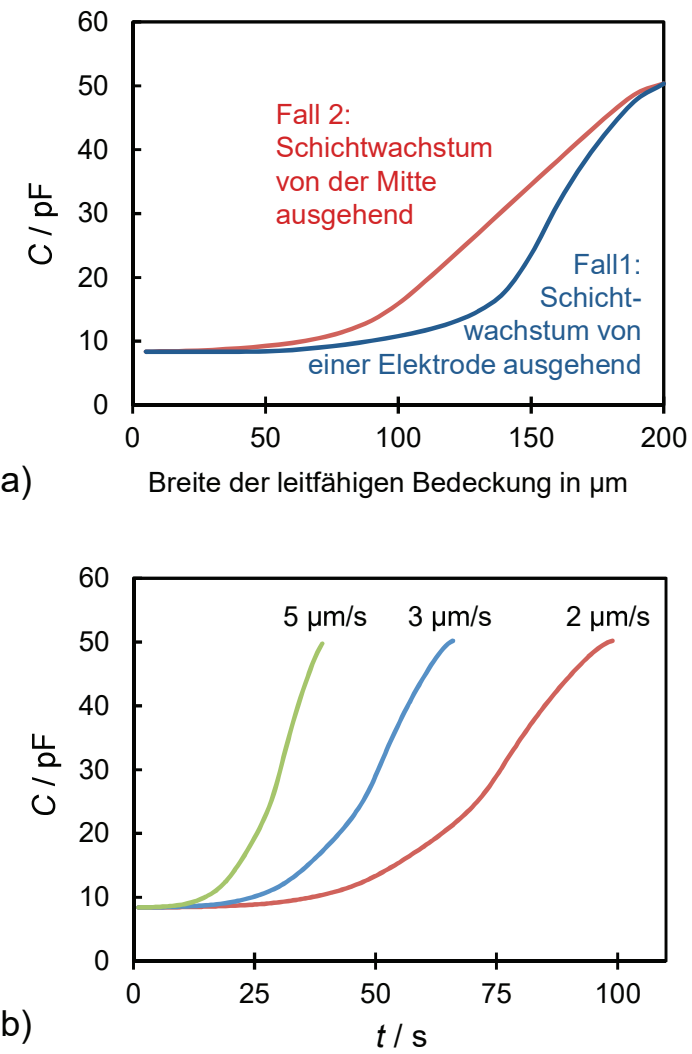

Abb. 3: a) Simulation der Kapazität einer Interdigitalelektrodenstruktur in $A b$ hängigkeit der Bedeckung mit leitfähigem Material für zwei Grenzfälle: Fall 1 zeigt die Kapazitätsänderung falls die Schicht 
von einer Elektrode ausgehend wächst; Fall 2 zeigt die Änderungen falls die Schicht von der Mitte heraus wächst. b) Simulation der Kapazitätsänderung über dem zeitlichen Verlauf des Schicht-wachstums für unterschiedliche Wachstumsraten.

Nimmt man weiter an, dass die Bedeckung der Sensoroberfläche durch sich anlagernden Ruß entsteht, dann kann man ebenfalls einen zu erwartenden Signalverlauf für die Zeit eines Messzyklus berechnen. Hierzu wird angenommen, dass die leitfähige Bedeckung mit einer konstanten Rate wächst. $\mathrm{Da}$ die Anlagerung von Ruß zufällig erfolgt, werden diese Rechnungen für beide oben beschriebenen Grenzfälle durchgeführt und der Mittelwert aus beiden Verläufen aufgetragen (Abbildung 3b). Für eine niedrige Rußkonzentration im Abgas wird angenommen, dass die Wachstumsrate klein ist (hier: 2 $\mu \mathrm{m} / \mathrm{s}$ ), höhere Wachstumsraten beschreiben höhere Rußkonzentrationen, d.h. mehrere Partikel erreichen die Sensoroberfläche in der gleichen Zeit. Es wird deutlich, dass bei steigender Rußkonzentration (d.h. höheren Wachstumsraten) einerseits die Zeit bis zum steilen Anstieg verkürzt wird, andererseits sich aber auch die Kapazitätsänderung (Steigung der Kurve) erhöht.

\section{Vergleich mit realen Messungen}

Um diese Überlegungen im realen Versuch nachweisen zu können wurden zunächst kapazitive Rußsensoren hergestellt. Die Elektrodenstruktur wurde aus $\mathrm{Pt}$ im Siebdruckverfahren auf einem $\mathrm{Al}_{2} \mathrm{O}_{3}$-Substrat realisiert. Die Interdigital-Elektroden besitzen $100 \mu \mathrm{m}$ breite Finger mit $100 \mu \mathrm{m}$ Abständen. Der Elektrodenbereich (siehe Abb. 1a) wurde mit einer $\mathrm{Al}_{2} \mathrm{O}_{3}$-Schicht (8 $\mathrm{\mu m}$ dick) auf der Oberfläche isoliert. Um eine dünne, aber komplett dichte Schicht zu erzeugen, die außerdem hochtemperaturstabil ist, wurde dafür die neuartige Aerosol-Beschichtung angewendet $[6,7]$. Hierbei können keramische Schichten in einem kalten Prozess (d.h. ohne Sintervorgang) direkt aus dem Pulver abgeschieden werden. Auf der Rückseite des Sensors wurde eine Dickschicht-Heizerstruktur integriert, mit der es möglich ist, den Sensor zu „regenerieren“. Das Aufheizen des Bauteils auf ca. $600{ }^{\circ} \mathrm{C}$ führt zum Abbrand des abgelagerten Rußes. So kann ein neuer Messzyklus gestartet werden.

Die Messung der Kapazität des Sensorelements erfolgte bei $10 \mathrm{kHz}$ im realen Abgas eines Dieselmotors bei $1000 \mathrm{U} / \mathrm{min}$ und $25 \%$ Last. Die Veränderung der Rußkonzentration für verschiedene Messungen erfolgte durch Variation des Ladedrucks $p_{\mathrm{L}}$ am Motor. Durch den niedrigeren Ladedruck sind auf

Grund

inhomogenerer

Verbrennungsbedingungen höhere Rußkonzentrationen im Abgas zu erwarten. Dies kann durch gleichzeitige Messung der Rußmenge im Abgas mittels eines kommerziellen Partikelmessgerätes (PEGASOR-Sensor) verifiziert werden (Tabelle 1).

Tab. 1: Zusammenhang der Partikelmenge im Abgas in Abhängigkeit des Ladedrucks bei $1000 \mathrm{U} / \mathrm{min}$ und $25 \%$ Last.

\begin{tabular}{|c|c|c|}
\hline $\begin{array}{c}p_{\mathrm{L}} / \\
\text { bar }\end{array}$ & $\begin{array}{c}\text { Partikel- } \\
\text { masse }\end{array}$ & Partikelanzahl \\
\hline 1,22 & $9 \mathrm{mg} / \mathrm{m}^{3}$ & $34,2 \times 10^{6} / \mathrm{m}^{3}$ \\
\hline 1,17 & $22 \mathrm{mg} / \mathrm{m}^{3}$ & $84,8 \times 10^{6} / \mathrm{m}^{3}$ \\
\hline 1,12 & $39 \mathrm{mg} / \mathrm{m}^{3}$ & $152,4 \times 10^{6} / \mathrm{m}^{3}$ \\
\hline
\end{tabular}

Mit der Erniedrigung des Ladedrucks ergeben sich außerdem leichte Änderungen im Abgasmassenstrom (Erniedrigung von 80 bis ca. $75 \mathrm{~kg} / \mathrm{h}$ ), Änderungen im Lambda (Erniedrigung von 1,38 bis 1,25) und Änderungen in der Abgastemperatur (Erhöhung von 270 bis ca. $315^{\circ} \mathrm{C}$ ).

Abbildung 4 zeigt drei SensorBeladungszyklen, die mit diesen unterschiedlichen Motor-Betriebszuständen erreicht wurden. Zur besseren Übersicht wurden alle drei Messungen auf eine gemeinsame Startzeit $(t=0)$ bezogen, die jeweils den Start der Beladung mit Ruß angibt.

Man erkennt leicht, dass bei Erniedrigung des Ladedrucks die Messkurve nach links verschoben wird und gleichzeitig der Kapazitätsanstieg steiler wird. Der Sättigungswert der Kapazität nimmt ebenfalls leicht zu, was aber auch mit der damit einhergehenden Steigerung der Abgastemperatur zusammenhängen kann.

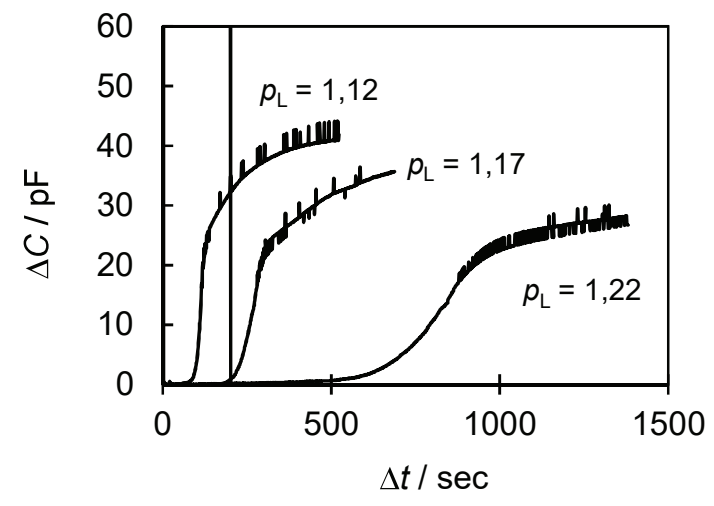

Abb. 4: Messungen eines kapazitiven Rußsensors im Abgasstrom eines 2,1 I Dieselmotors bei 1000 U/min und $25 \%$ Last. Der Einspritzdruck lag bei 550 bar. Unterschiedliche Rußkonzentrationen wurden durch Variation des Ladedrucks erreicht (1,22 - 1,17 bar) 


\section{Zusammenfassung und Ausblick}

Die Simulation der Kapazitätsänderung eines neuartigen kapazitiven Rußsensors zeigt im Prinzip einen sehr ähnlichen Verlauf, wie er auch bei realen Messungen im Dieselabgas eines Verbrennungsmotors erreicht wird. Mit Hilfe der Berechnungen sollen Möglichkeiten zur Erhöhung der Sensitivität eines solchen Sensors ermittelt und technisch umgesetzt werden, um für den Anwendungsfall geringste Partikelkonzentrationen im Dieselabgas detektieren zu können.

\section{Literaturnachweis}

[1] M. Twigg, Cleaning the Air We Breathe Controlling Diesel Particulate Emissions from Passenger Cars. Platinum Metals Rev. 53 (2009) 27-34, doi: 10.1595/147106709X390977

[2] T. Ochs et al., Particulate Matter Sensor for On Board Diagnostics (OBD) of Diesel Particulate Filters (DPF). SAE Technical Paper 2010-010307 (2010).

[3] M. Feulner et al., Conductometric sensor for soot mass flow detection in exhausts of internal combustion engines. Sensors 15 (2015) 28796 28806; doi:10.3390/s151128796

[4] D. Grondin et al., Development of a particulate matter sensor for diesel engine. Procedia Engineering 120 (2015) 1237-1240, doi: 10.1016/j.proeng.2015.08.838

[5] G. Hagen et al., Capacitive Soot Sensor. Procedia Engineering 120 (2015) 241- 244, doi: 10.1016/j.proeng.2015.08.590

[6] D. Hanft et al., An Overview of the Aerosol Deposition Method: Process Fundamentals and New Trends in Materials Application. Journal of Ceramic Science and Technology 6 (2015) 147182, doi: 10.4416/JCST2015-00018

[7] M. Schubert et. al., Influence of Carrier Gas Composition on the Stress of $\mathrm{Al}_{2} \mathrm{O}_{3}$ Coatings Prepared by the Aerosol Deposition Method. Materials, 7 (2014) 5633-5642,

doi: $10.3390 / \mathrm{ma} 7085633$ 